\title{
MARKOV MODEL OF TEMPERATURE CHANGES IN MOTOR VEHICLES BRAKE DISCS
}

\author{
D. Perczyński", K. Migawa*, K. Peszynski", L. Knopik**
}

\begin{abstract}
The developed mathematical model is to allow to diagnose the state of fitness of individual vehicle brake friction nodes in real time In this work, it is assumed that the braking force and ambient temperature of the brake disc are the implementation of certain random processes over time. The purpose of the work is to describe the processes of changing the braking force and changing the temperature of the brake disc. It has been shown that an adequate model of these changes may be the semi-Markov process.
\end{abstract}

Keywords: random process, semi-Markov process, alternate process

\section{Introduction}

The motor vehicles braking systems during their operation show the occurrence of operating conditions defined as incorrect. The irregularity is determined, for example, as blocking the wheel, occurrence of the difference in braking force on the axle greater than $30 \%$, and in extreme cases, no braking force. Higher vehicle speeds and reducing the dimensions of the brake pair (disc - brake pad) forces the use of higher pressures between the brake pad and the disc and also increases the thermal load of the braking system. Increased requirements imposed on brake systems force not only periodical monitoring of friction pair condition, but also continuous monitoring of the braking system's fitness.

During braking of the truck in the brake discs as a result of friction increases the temperature. The rate of temperature increase depends primarily on the braking force. After the braking period, the temperature drops to ambient temperature. However, an event is possible in which the brake discs do not reach ambient temperature because subsequent braking occurs. This fact shows the basic postulate that should be taken into account in the description of temperature changes during braking. During braking, there are random events such as: moment of braking start, moment of braking end, duration of braking, braking force. All of the above-mentioned events occur randomly during observation, therefore, stochastic processes are proposed as models of changes. In this work, a semi-Markov process is considered as a model of changes in braking force and temperature changes.

\section{Change of braking force as a function of time}

In order to ensure the possibility of using semi-Markov processes, braking force is quantified using $n$ discrete values $\{0,1,2,3, \ldots, n\}$, see Fig. 1 . If $X(t)$ is the braking force at the moment $t$, equality $X(t)=n$ means that at time $t$ the braking is performed with the highest value of. If $X(t)=0$ means that

Assist. Prof. Daniel Perczyński, PhD.: UTP University of Science and Technology (Poland), Faculty of Mechanical Engineering, Al. prof. S. Kaliskiego 7, 85-796 Bydgoszcz; PL, daniel.perczynski@utp.edu.pl

* Assoc. Professor Klaudiusz Migawa, PhD., UTP University of Science and Technology (Poland), Faculty of Mechanical Engineering Al. Prof. S. Kaliskiego 7, Bydgoszcz; PL, klaudiusz.migawa@utp.edu.p

* Assoc. Professor Kazimierz Peszyński, PhD., UTP University of Science and Technology (Poland), Faculty of Mechanical Engineering Al. Prof. S. Kaliskiego 7, Bydgoszcz; PL, kazimierz.peszynski@utp.edu.pl

** Assoc. Prof. Leszek Knopik, PhD.: UTP University of Science and Technology, Faculty of Management, Fordońska 430, 85-790 Bydgoszcz, PL, leszek.knopik@utp.edu.pl 
the driver does not brake. In general, if $X(t)=i, i \in\{0,1,2,3, \ldots, n\}$, then the driver performs braking with force $i$.

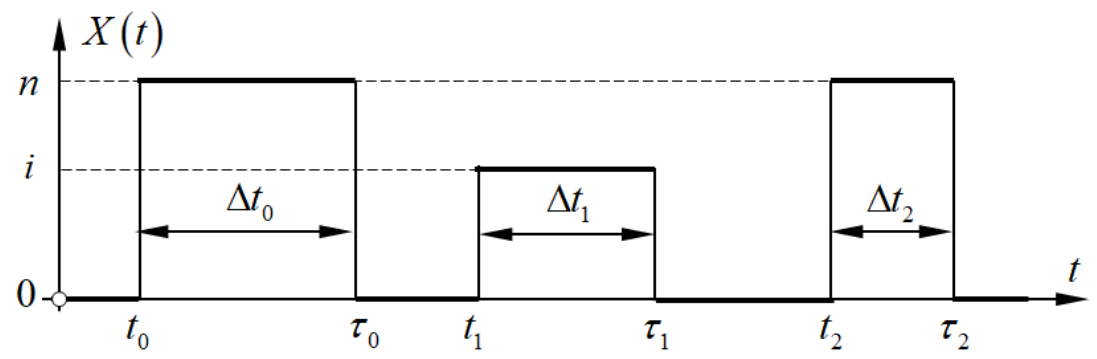

Fig. 1: Implementation of the braking process

To define a semi-Markov process $X(t)$, one must create (or identify) the initial distribution of the character process:

$$
P\{X(0)=i\}=p_{i}, \text { where } i=0,1,2, \ldots, n,
$$

and

$$
\sum_{i=0}^{n} p_{i}=1
$$

To determine the semi-Markov process, it is necessary to determine the elements of the process core. The core of the process is a function matrix of the form:

$$
\mathbf{Q}(t)=\left[Q_{i j}(t): i, j \in S\right],
$$

where

$$
Q_{i j}(t)=P\left(X\left(\tau_{n+1}-\tau_{n}\right) \leq t, X\left(\tau_{n+1}\right)=j \mid X\left(\tau_{n}\right)=i\right), t \geq 0 .
$$

The sequence of random variables is a uniform Markov chain with probabilities of transition (state changes):

$$
p_{i j}=P\left(X\left(\tau_{n+1}\right)=j \mid X\left(\tau_{n}\right)=i\right)=\lim _{t \rightarrow \infty} Q_{i j}(t) .
$$

Function:

$$
G_{i}(t)=P\left(T_{i} \leq t\right)=P\left(\tau_{n+1}-\tau_{n} \leq t \mid X\left(\tau_{n}\right)=i\right)=\sum_{j \in S} Q_{i j}(t), t \geq 0
$$

is a cumulative distribution of time $T_{i}$ in the state $S_{i}$.

Conditional cumulative distribution function of the random variable $T_{i}$, of time spent in the state $S_{i}$ provided that the previous state was a state $S_{j}$ is expressed by a formula

$$
F_{i j}(t)=P\left(\tau_{n+1}-\tau_{n} \leq t \mid X\left(\tau_{n}\right)=i, X\left(\tau_{n+1}\right)=j\right)=\frac{Q_{i j}(t)}{p_{i j}}
$$

Based on the above relationships, it is known that:

$$
Q_{i j}(t)=p_{i j} \cdot F_{i j}(t)
$$

In a special case, it is assumed that $X(t)$ takes two values: 0 when there is no braking at time $t$ and a value of 1 when there is braking at time $t$. If the braking time and the time without braking have exponential distributions, $X(t)$ is a Markov alternating process. If one of the above mentioned times or both times have a distribution different from the exponential distribution, the $X(t)$ process is a two-state process of semi-Markov. 
Let $t_{0}, t_{1}, t_{2}, \ldots$ indicate the start times of braking, however $\tau_{0}, \tau_{1}, \tau_{2}, \ldots$ indicate the appropriate time for stopping the braking, then $\Delta t_{i}$ determine the duration of the $i$-th braking. An exemplary implementation of the alternation process is shown in Fig. 1.

Braking in the $i$-th interval starts at time $t_{\mathrm{i}}$, ends at time $\tau_{\mathrm{i}}$, whereas the temperature lowering process will take place in the time range $\left(\tau_{i}, t_{i+1}\right)$. It is possible that the driver will start the next braking before the brake discs have reached the ambient temperature. In this case, the temperature of the brake discs will increase from a value higher than the ambient temperature. The course of the temperature value in the cycle $(i+1)$ therefore depends on the temperature value at the time $\tau_{\mathrm{i}}$.

\section{Process of car discs temperature changes}

Implementation of the braking process generates a process of changing the temperature of the brake discs during driving of the car.

The process $Y(t)$ models temperature changes over time, if $Y(t)=v$ that means that at the time $t$ the temperature of the brake discs is $v$. It can be assumed that the process $Y(t)$ is a semi-Markov process with an infinite number of states. More precisely, with a finite number of states. The implementation $Y(t)$ of the process is shown in Figure 2.

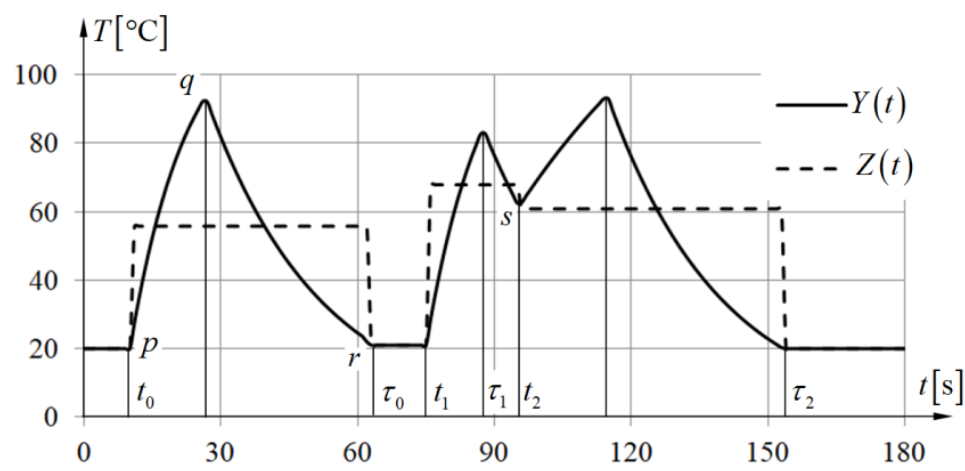

Fig. 2: Implementation of the process $Y(t)$ and the corresponding process $Z(t)$

The numerical description of the curves of one braking cycle (increase and decrease in temperature) was studied in the works (Perczyński et al., 2016 and Szews et al., 2017). In the study of the nature of this relationship, the dependence of the maximum temperature on the braking force and on the length of the braking time is important. This relationship can be helpful in simplifying of the process $Z(t)$ creation.

Based on the properties of the process $Y(t)$, a new process can be defined $Z(t)$. Let $p$ be the moment of start of braking, $q$ is the moment of ending this braking and $r$ is the moment when the brakes get the ambient temperature. It is possible that the subsequent braking has started at the time $s$, such that $s<r$, before the brakes get the ambient temperature. If $s \geq r$ then, $Z(t)$ is the mean value of the function in the interval, whereas in the case of $s<r$ the value $Z(t)$ is defined as the average of the function in the interval $[p, s]$. The average value of the process $Y(t)$ in the interval $[p, r]$ is expressed by the formula:

$$
Z(t)=\frac{1}{r-p} \int_{p}^{r} Y(w) d w,
$$

where $t \in[p, r]$. This the integral can be approximately calculated by formula:

$$
\hat{Z}(t)=\frac{1}{r-p} \sum_{i=1}^{n} Y\left(w_{i}\right), .
$$


where $w_{i}=p+(i-1)(r-p) /(n-1) \quad i=1,2, \ldots, n$.

Such a defined process $Z(t)$ is a constant process in intervals. From the physical conditions it appears that it can be assumed that the process $Z(t)$ is a semi-Markov process. An example of the implementation of the process $Z(t)$ is shown in Fig. 2.

\section{An example of using the semi-Markov process}

The example below analyzes processes $Y(t)$ and $Z(t)$, Fig 3. Similarly as for a process $X(t)$, the set of process values can be grouped into classes $\{0,1,2, \ldots, m\}$. Then the process $Z(t)$ will take a finite number of values. The condition $Z(t)=0$ means that the discs have a temperature close to the ambient temperature.

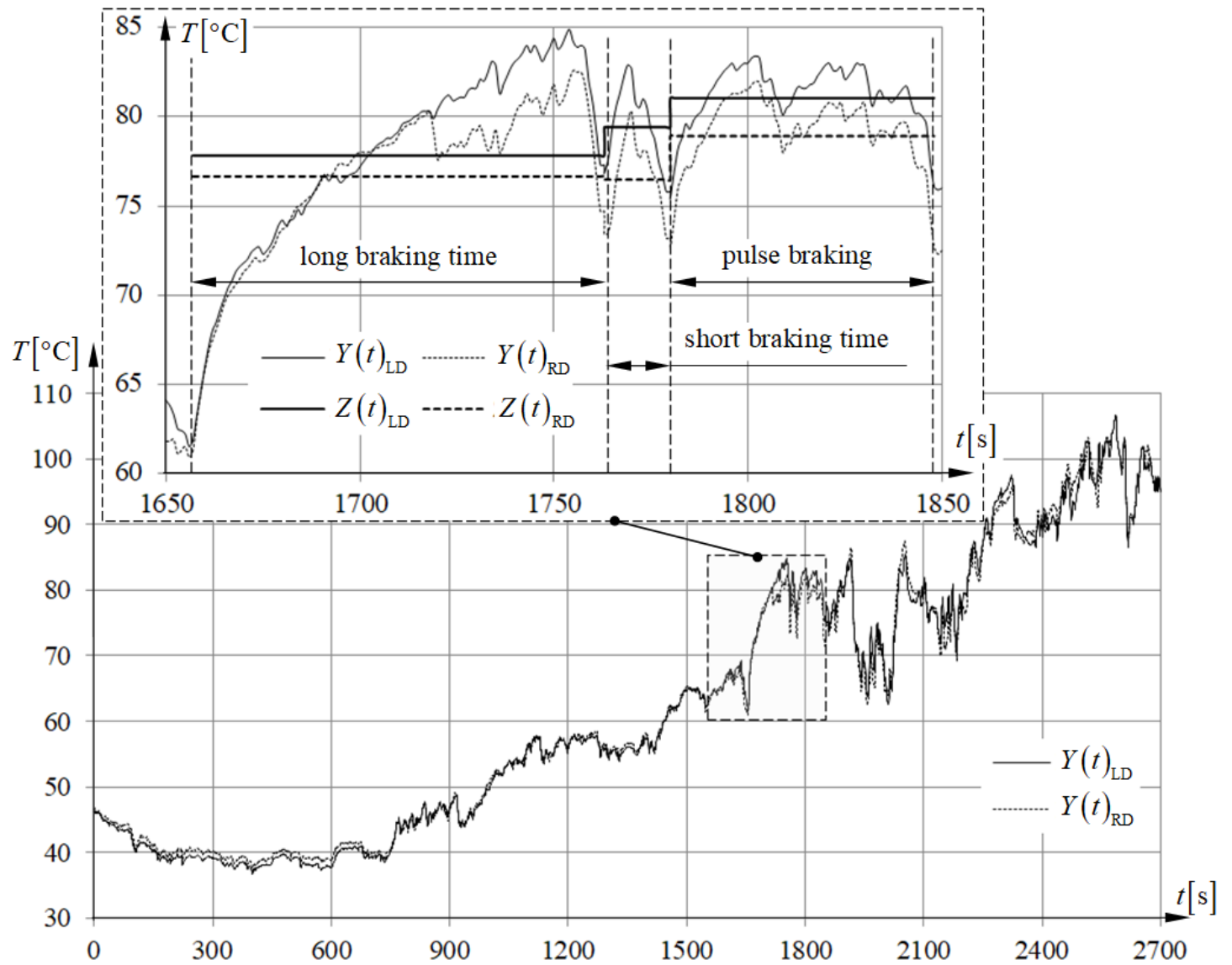

Fig. 3: Real implementation of processes $Y(t)$ and $Z(t)-\mathrm{LD}$ disk of left wheel, $\mathrm{RD}$ disk of right wheel.

\section{Conclusions}

In the paper as a model of changes in time of braking force and temperature, a homogeneous semiMarkov process is considered. The function $Z(t)$ presented above is to enable, in the next stage, to develop a form of a criterion function to assess the fitness of friction nodes of motor vehicles.

\section{References}

Perczyński D., Peszyński K., Knopik L., Wawrzyniak S. (2016) Temperature analysis in the vicinity of triaxial trailer disc brake, Engineering Mechanics 2016, IT AS CR, Prague, pp. 450-453.

Szews M., Perczyński D., Knopik L., Wawrzyniak S. (2017) Temperature analysis dependence in the vicinity of the brake disk, Engineering Mechanics 2017, Brno University of Technology, Brno, pp. 962-965. 